\title{
Rhodium-Catalyzed Addition/Ring-Opening Reaction of Arylboronic Acids with Cyclobutanones
}

\author{
Takanori Matsuda, Masaomi Makino, and Masahiro Murakami* \\ Department of Synthetic Chemistry and Biological Chemistry, Kyoto University, Katsura, \\ Kyoto 615-6510, Japan
}

\section{Supporting Information}

General. All reactions were carried out with standard Schlenk techniques under an argon atmosphere. NMR spectra were recorded on a Varian Mercury $400\left({ }^{1} \mathrm{H}\right.$ at 400.44 $\mathrm{MHz})$ or a Varian Gemini $2000\left({ }^{1} \mathrm{H}\right.$ at $300.77 \mathrm{MHz}$ and ${ }^{13} \mathrm{C} \mathrm{NMR}$ at $\left.75.46 \mathrm{MHz}\right)$. Chemical shifts are reported in $\delta \mathrm{ppm}$ referenced to a residual chloroform $\left(\delta 7.26\right.$ for ${ }^{1} \mathrm{H}$, $\delta 77.0$ for ${ }^{13} \mathrm{C}$ ). Infrared spectra were recorded on a Mettler-Toledo ReactIR 4000. High resolution mass spectra were recorded on a JEOL JMS-SX102A. Preparative thin-layer chromatography was performed with silica Gel $60 \mathrm{PF}_{254}$ (Merck).

Materials. Cyclobutanones $\mathbf{1 a}-\mathbf{c}^{1}$ and $\mathbf{1} \mathbf{d}^{2,3}$ were prepared according to the literature procedures. $p$-Tolylboronic acid pinacol ester $\mathbf{2}^{\prime} \mathbf{b}$ was prepared by esterification of $p$ tolylboronic acid with pinacol in methylene chloride. 1,4-Dioxane was distilled over $\mathrm{LiAlH}_{4}$ before using for catalytic reactions. All other commercially available chemical resources were used without further purifications.

\section{Rh-Catalyzed Reaction of Cyclobutanones 1 and Arylboronic Acids 2 (Table 1)}

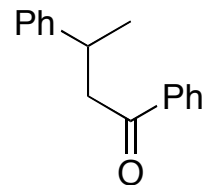

1,3-Diphenylbutan-1-one (3ac) $\quad[1533-20-6] .{ }^{4}$ To a mixture of $\mathrm{Rh}(\mathrm{acac})\left(\mathrm{C}_{2} \mathrm{H}_{4}\right)_{2}(6.46 \mathrm{mg}$, $0.025 \mathrm{mmol}$ ), 2c (183 mg, $1.50 \mathrm{mmol}$ ), and $\mathrm{Cs}_{2} \mathrm{CO}_{3}$ (163 mg, $0.50 \mathrm{mmol}$ ) were added 1,4dioxane $(1.0 \mathrm{~mL}), \mathrm{P}(t-\mathrm{Bu})_{3}(10.1 \mathrm{mg}, 0.050 \mathrm{mmol})$, and $1 \mathrm{a}(73.1 \mathrm{mg}, 0.50 \mathrm{mmol})$. After being stirred for $24 \mathrm{~h}$ at $100{ }^{\circ} \mathrm{C}$, the reaction mixture was extracted with ether $(50 \mathrm{~mL})$, 
washed with brine, and dried over $\mathrm{MgSO}_{4}$. Bulb-to-bulb distillation $\left(220{ }^{\circ} \mathrm{C} / 1-2 \mathrm{mmHg}\right)$ gave 3ac (106.5 mg, $95 \%$ ) as a colorless oil. 1a: ${ }^{13} \mathrm{C}$ NMR $\delta 21.7,35.3,46.8,126.2,126.8$, $127.9,128.1,128.4,132.9,137.1,146.5,198.9$.

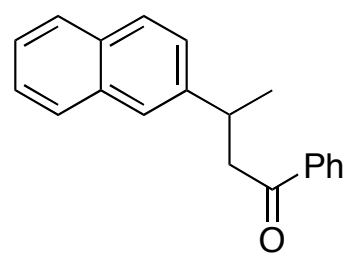

3-(2-Naphtyl)-1-phenylbutan-1-one (3bc). To a mixture of $\mathrm{Rh}(\mathrm{acac})\left(\mathrm{C}_{2} \mathrm{H}_{4}\right)_{2}(6.46 \mathrm{mg}$, $0.025 \mathrm{mmol}), 2 \mathrm{c}(181 \mathrm{mg}, 1.49 \mathrm{mmol})$, and $\mathrm{Cs}_{2} \mathrm{CO}_{3}(163 \mathrm{mg}, 0.50 \mathrm{mmol})$ were added 1,4dioxane $(1.0 \mathrm{~mL}), \mathrm{P}(t-\mathrm{Bu})_{3}(10.1 \mathrm{mg}, 0.050 \mathrm{mmol})$, and $\mathbf{1 b}(98 \mathrm{mg}, 0.51 \mathrm{mmol})$. After being stirred for $24 \mathrm{~h}$ at $100{ }^{\circ} \mathrm{C}$, the reaction mixture was filtered through a pad of Florisil ${ }^{\circledR}$ (ether). The filtrate was evaporated and the residue was purified by preparative thin-layer chromatography of silica gel (hexane:AcOEt $=8: 1)$ to afford 3bc $(102 \mathrm{mg}, 78 \%) .3 \mathbf{b c}$ : ${ }^{1} \mathrm{H}$ NMR $(300 \mathrm{MHz}) \delta 1.49(\mathrm{~d}, J=7.0 \mathrm{~Hz}, 3 \mathrm{H}), 3.32(\mathrm{dd}, J=16.5,8.1 \mathrm{~Hz}, 1 \mathrm{H}), 3.45(\mathrm{dd}, J=$ 16.5, 5.7 Hz, 1H), 3.75 (sext, $J=7.0 \mathrm{~Hz}, 1 \mathrm{H}), 7.40-7.50(\mathrm{~m}, 5 \mathrm{H}), 7.50-7.58(\mathrm{~m}, 1 \mathrm{H}), 7.70-$ $7.73(\mathrm{~m}, 1 \mathrm{H}), 7.77-7.84(\mathrm{~m}, 3 \mathrm{H}), 7.93-7.99(\mathrm{~m}, 2 \mathrm{H}) ;{ }^{13} \mathrm{C}$ NMR $\delta$ 21.9, 35.6, 46.9, 124.9, 125.3, 125.6, 125.9, 127.5, 127.6, 128.0, 128.1, 128.5, 132.2, 132.9, 133.5, 137.1, 144.0, 198.9; HRMS (EI) calcd for $\mathrm{C}_{20} \mathrm{H}_{18} \mathrm{O} 274.1358$, found 274.1358.

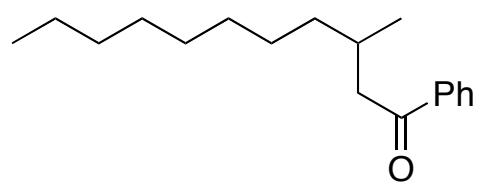

3-Methyl-1-phenylundecan-1-one (3cc). According to the procedure described for 3bc, $3 \mathbf{c c}(98 \mathrm{mg}, 75 \%)$ was prepared from $1 \mathrm{c}(92 \mathrm{mg}, 0.50 \mathrm{mmol})$ and $\mathbf{2 c}(183 \mathrm{mg}, 1.50 \mathrm{mmol})$. 3cc: IR (neat) 2925, 1688, 1598, 1449, 1364, 1281, 1214, 1003, 751, 691 $\mathrm{cm}^{-1}$; ${ }^{1} \mathrm{H}$ NMR (400 MHz) $\delta 0.87(\mathrm{t}, J=6.8 \mathrm{~Hz}, 3 \mathrm{H}), 0.94(\mathrm{~d}, J=6.4 \mathrm{~Hz}, 3 \mathrm{H}), 1.20-1.42(\mathrm{~m}, 14 \mathrm{H}), 2.09-$ $2.22(\mathrm{~m}, 1 \mathrm{H}), 2.74(\mathrm{dd}, J=15.6,8.0 \mathrm{~Hz}, 1 \mathrm{H}), 2.94(\mathrm{dd}, J=15.6,5.6 \mathrm{~Hz}, 1 \mathrm{H}), 7.42-7.48(\mathrm{~m}$, $2 \mathrm{H}), 7.51-7.57(\mathrm{~m}, 1 \mathrm{H}), 7.92-7.97(\mathrm{~m}, 2 \mathrm{H}) ;{ }^{13} \mathrm{C} \mathrm{NMR} \delta 14.0,19.9,22.6,26.9,29.2,29.5$, $29.7,31.8,37.1,45.9,128.1,128.5,132.8,137.5,200.5$ [one carbon signal is missing]; HRMS (EI) calcd for $\mathrm{C}_{18} \mathrm{H}_{28} \mathrm{O}$ 260.2140, found 260.2145; Anal. Calcd. for $\mathrm{C}_{18} \mathrm{H}_{28} \mathrm{O}$ : C, 
83.02; H, 10.84. Found: C, 83.30; H, 10.67.<smiles>CCC(C(=O)c1ccccc1)c1ccccc1</smiles><smiles>O=C(CCCc1ccccc1)c1ccccc1</smiles>

\section{1,2-Diphenylbutan-1-one (3dc) [16282-16-9] and 1,4-Diphenylbutan-1-one (3'dc)} [5407-91-0]. According to the procedure described for 3bc, a 77:23 mixture of 3da and 3'da (25 mg, 52\%) was prepared from $1 d$ (31 mg, $0.21 \mathrm{mmol})$ and $\mathbf{2 c}(78 \mathrm{mg}, 0.64 \mathrm{mmol})$. 3dc: ${ }^{1} \mathrm{H}$ NMR $(400 \mathrm{MHz}) \delta 0.91(\mathrm{t}, J=7.2 \mathrm{~Hz}, 3 \mathrm{H}), 1.86(\mathrm{dq}, J=13.8,7.3 \mathrm{~Hz}, 1 \mathrm{H}), 2.21$ $(\mathrm{dq}, J=13.8,7.3 \mathrm{~Hz}, 1 \mathrm{H}), 4.45(\mathrm{t}, J=7.2 \mathrm{~Hz}, 1 \mathrm{H}), 7.17-7.22(\mathrm{~m}, 1 \mathrm{H}), 7.25-7.32(\mathrm{~m}, 4 \mathrm{H})$, 7.36-7.42 (m, 2H), 7.45-7.50 (m, 1H), 7.94-7.98 (m, 2H); ${ }^{13} \mathrm{C}$ NMR $\delta 12.3,27.1,55.5$, 126.9, 128.2, 128.4, 128.6, 128.8, 132.7, 137.0, 139.6, 200.0. 3'dc: ${ }^{1} \mathrm{H}$ NMR (400 MHz) $\delta$ 2.09 (quint, $J=7.4 \mathrm{~Hz}, 2 \mathrm{H}), 2.73(\mathrm{t}, J=7.6 \mathrm{~Hz}, 2 \mathrm{H}), 2.98(\mathrm{t}, J=7.2 \mathrm{~Hz}, 2 \mathrm{H}), 7.17-7.24(\mathrm{~m}$, $2 \mathrm{H}), 7.24-7.32(\mathrm{~m}, 2 \mathrm{H}), 7.42-7.48(\mathrm{~m}, 2 \mathrm{H}), 7.52-7.57(\mathrm{~m}, 1 \mathrm{H}), 7.90-7.94(\mathrm{~m}, 2 \mathrm{H}) ;{ }^{13} \mathrm{C}$ NMR $\delta 25.7,35.2,37.7,125.9,128.0,128.4,128.48,128.52,132.9,137.0,141.6,200.0$.

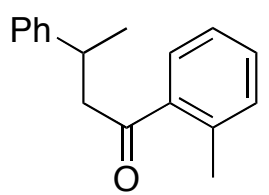

1-(2-Methylphenyl)-3-phenylbutan-1-one (3aa). According to the procedure described for 3bc, 3aa (86 mg, 72\%) was prepared from 1a $(73 \mathrm{mg}, 0.50 \mathrm{mmol})$ and $2 \mathbf{a}(203 \mathrm{mg}$, $1.49 \mathrm{mmol}) .3 \mathrm{aa}:{ }^{1} \mathrm{H}$ NMR (400 MHz) $\delta 1.34(\mathrm{~d}, J=6.8 \mathrm{~Hz}, 3 \mathrm{H}), 2.37$ (s, 3H) $3.12(\mathrm{dd}, J$ $=16.8,8.4 \mathrm{~Hz}, 1 \mathrm{H}), 3.23(\mathrm{dd}, J=16.4,6.4 \mathrm{~Hz}, 1 \mathrm{H}), 3.46$ (sext, $J=6.8 \mathrm{~Hz}, 1 \mathrm{H}), 7.17-7.40$ $(\mathrm{m}, 8 \mathrm{H}), 7.52-7.59(\mathrm{~m}, 1 \mathrm{H}) ;{ }^{13} \mathrm{C}$ NMR $\delta 20.7,21.9,35.8,49.9,125.5,126.2,126.9,128.1$, 128.5, 131.0, 131.8, 137.8, 138.4, 146.3, 203.6; HRMS (EI) calcd for $\mathrm{C}_{17} \mathrm{H}_{18} \mathrm{O} 238.1358$, found 238.1358. Anal. Calcd for $\mathrm{C}_{17} \mathrm{H}_{18} \mathrm{O}: \mathrm{C}, 85.67$; H, 7.61. Found: C, 85.69; H, 7.64.

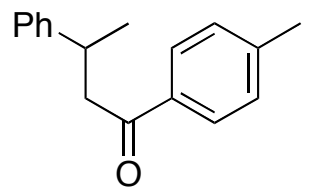

1-(4-Methylphenyl)-3-phenylbutan-1-one (3ab). According to the procedure described 
for 3bc, 3ab (98 mg, 82\%) was prepared from $\mathbf{1 a}(74 \mathrm{mg}, 0.50 \mathrm{mmol})$ and $\mathbf{2 b}(203 \mathrm{mg}$, $1.49 \mathrm{mmol}) .{ }^{1} \mathrm{H}$ NMR (400 MHz) $\delta 1.36(\mathrm{~d}, J=7.2 \mathrm{~Hz}, 3 \mathrm{H}), 2.42(\mathrm{~s}, 3 \mathrm{H}), 3.18(\mathrm{dd}, J=$ 16.2, 8.2 Hz, 1H), 3.30 (dd, $J=16.2,5.8 \mathrm{~Hz}, 1 \mathrm{H}$ ), 3.53 (sext, $J=7.0 \mathrm{~Hz}, 1 \mathrm{H}$ ), 7.18-7.40 $(\mathrm{m}, 7 \mathrm{H}), 7.84-7.92(\mathrm{~m}, 2 \mathrm{H}) ;{ }^{13} \mathrm{C}$ NMR $\delta 21.6,21.8,35.6,46.9,126.2,126.8,128.1,128.4$, 129.2, 134.6, 143.6, 146.6, 198.6; HRMS (EI) calcd for $\mathrm{C}_{17} \mathrm{H}_{18} \mathrm{O}$ 238.1358, found 238.1358 .

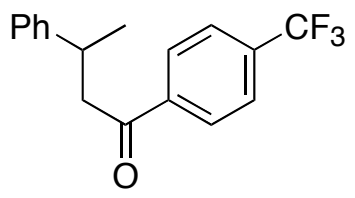

1-(4-Trifluoromethylphenyl)-3-phenylbutan-1-one (3ad). According to the procedure described for 3bc, 3ad (57 mg, 39\%) was prepared from 1a (76 mg, $0.52 \mathrm{mmol})$ and 2d (285 mg, $1.50 \mathrm{mmol}) .3 \mathrm{ad}:{ }^{1} \mathrm{H}$ NMR $(400 \mathrm{MHz}) \delta 1.37(\mathrm{~d}, J=6.8 \mathrm{~Hz}, 3 \mathrm{H}), 3.22(\mathrm{dd}, J=$ 16.4, 8.0 Hz, 1H), 3.35 (dd, $J=16.4,6.0 \mathrm{~Hz}, 1 \mathrm{H}), 3.52$ (sext, $J=6.9 \mathrm{~Hz}, 1 \mathrm{H}), 7.19-7.38$ $(\mathrm{m}, 5 \mathrm{H}), 7.69-7.77(\mathrm{~m}, 2 \mathrm{H}), 7.99-8.07(\mathrm{~m}, 2 \mathrm{H}) ;{ }^{13} \mathrm{C} \mathrm{NMR} \delta 21.9,35.5,47.3,123.6\left({ }^{1} J_{\mathrm{C}-\mathrm{F}}=\right.$ $272.5 \mathrm{~Hz}), 125.6\left({ }^{3} J_{\mathrm{C}-\mathrm{F}}=3.5 \mathrm{~Hz}\right), 126.4,126.8,128.3,128.6,134.2\left({ }^{2} J_{\mathrm{C}-\mathrm{F}}=32.8 \mathrm{~Hz}\right), 139.7$, 146.1, 198.0; HRMS (EI) calcd for $\mathrm{C}_{17} \mathrm{H}_{15} \mathrm{~F}_{3} \mathrm{O} 292.1075$, found 292.1078.

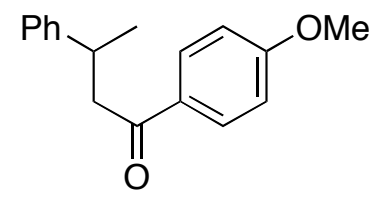

1-(4-Methoxyphenyl)-3-phenylbutan-1-one (3ae) [62557-94-2]..$^{5}$ According to the procedure described for 3bc, 3ae (54 mg, 42\%) was prepared from $1 \mathbf{a}$ (74 mg, $0.51 \mathrm{mmol})$ and 2e (228 mg, $1.50 \mathrm{mmol}$ ). 3ae: HRMS (EI) calcd for $\mathrm{C}_{17} \mathrm{H}_{18} \mathrm{O}_{2}$ 254.1307, found 254.1309 .

\section{Rh-Catalyzed Ring-Opening of Cyclobutanol 4aa (Scheme 2)}

A mixture of $\mathrm{Rh}(\mathrm{acac})\left(\mathrm{C}_{2} \mathrm{H}_{4}\right)_{2}(6.5 \mathrm{mg}, 0.025 \mathrm{mmol}), \mathrm{P}(t-\mathrm{Bu})_{3}(10 \mathrm{mg}, 0.050 \mathrm{mmol})$, $\mathrm{Cs}_{2} \mathrm{CO}_{3}(163 \mathrm{mg}, 0.50 \mathrm{mmol})$, and $4 \mathbf{a a}(115 \mathrm{mg}, 0.48 \mathrm{mmol})$ in 1,4-dioxane $(1.0 \mathrm{~mL})$ were stirred at $100{ }^{\circ} \mathrm{C}$ for $48 \mathrm{~h}$. The reaction mixture was extracted with ether $(50 \mathrm{~mL})$, washed with brine, dried over $\mathrm{MgSO}_{4}$. Concentration under reduced pressure afforded a mixture of 
3aa and 4aa (108 $\mathrm{mg}, 94 \%)$. The yield of 3aa was estimated to be $89 \%$ by ${ }^{1} \mathrm{H}$ NMR.

\section{Rh-Catalyzed Reaction of 3-Phenylcyclobutanone 1a with p-Tolylboronic Acid Pinacol Ester $2^{\prime} \mathrm{b}$ in $\mathrm{D}_{2} \mathrm{O}$ (Scheme 3).}

A mixture of $\mathrm{Rh}(\mathrm{acac})\left(\mathrm{C}_{2} \mathrm{H}_{4}\right)_{2}(6.5 \mathrm{mg}, 0.025 \mathrm{mmol}), \mathrm{P}(t-\mathrm{Bu})_{3}(10 \mathrm{mg}, 0.050 \mathrm{mmol}), 1 \mathrm{a}(72$ $\mathrm{mg}, 0.49 \mathrm{mmol})$, and $\mathbf{2}^{\prime} \mathbf{b}(330 \mathrm{mg}, 1.50 \mathrm{mmol})$ in 1,4-dioxane/ $\mathrm{D}_{2} \mathrm{O}(1: 1,2.0 \mathrm{~mL})$ were heated at $100{ }^{\circ} \mathrm{C}$ for $24 \mathrm{~h}$. The reaction mixture was extracted with ether $(50 \mathrm{~mL})$ and evaporated. The residue was purified by preparative thin-layer chromatography on silica gel (hexane:AcOEt = 8:1) to afford 3ab- $d$ as a diastereomeric mixture $(83 \% ; 92 \%$ D, 64:36 ratio by ${ }^{1} \mathrm{H}$ NMR). 3ab- $d:{ }^{1} \mathrm{H}$ NMR (400 MHz) $\delta 1.32$ (d, $J=6.8 \mathrm{~Hz}$, major $\left.3 \mathrm{H}\right), 1.33(\mathrm{~d}, J$ $=6.8 \mathrm{~Hz}$, minor $3 \mathrm{H}), 2.40(\mathrm{~s}, 3 \mathrm{H}), 3.13(\mathrm{dt}, J=8.4,2.3 \mathrm{~Hz}$, minor $1 \mathrm{H}), 3.23(\mathrm{dt}, J=5.2$, $2.3 \mathrm{~Hz}$, major $1 \mathrm{H}$ ), 3.49 (quint, $J=6.8 \mathrm{~Hz}, 1 \mathrm{H}), 7.16-7.32(\mathrm{~m}, 8 \mathrm{H}), 7.81-7.85(\mathrm{~m}, 2 \mathrm{H}) ;{ }^{13} \mathrm{C}$ NMR $\delta$ 21.6、 21.8 (major), 21.9 (minor), 35.6, $46.5\left({ }^{1} J_{\mathrm{C}-\mathrm{D}}=76.1 \mathrm{~Hz}\right), 126.2,126.8,128.2$, 128.4, 129.2, 134.6, 143.7, 146.6, 198.7; HRMS (EI) calcd for $\mathrm{C}_{17} \mathrm{H}_{17} \mathrm{DO} 239.1419$, found 239.1419. 


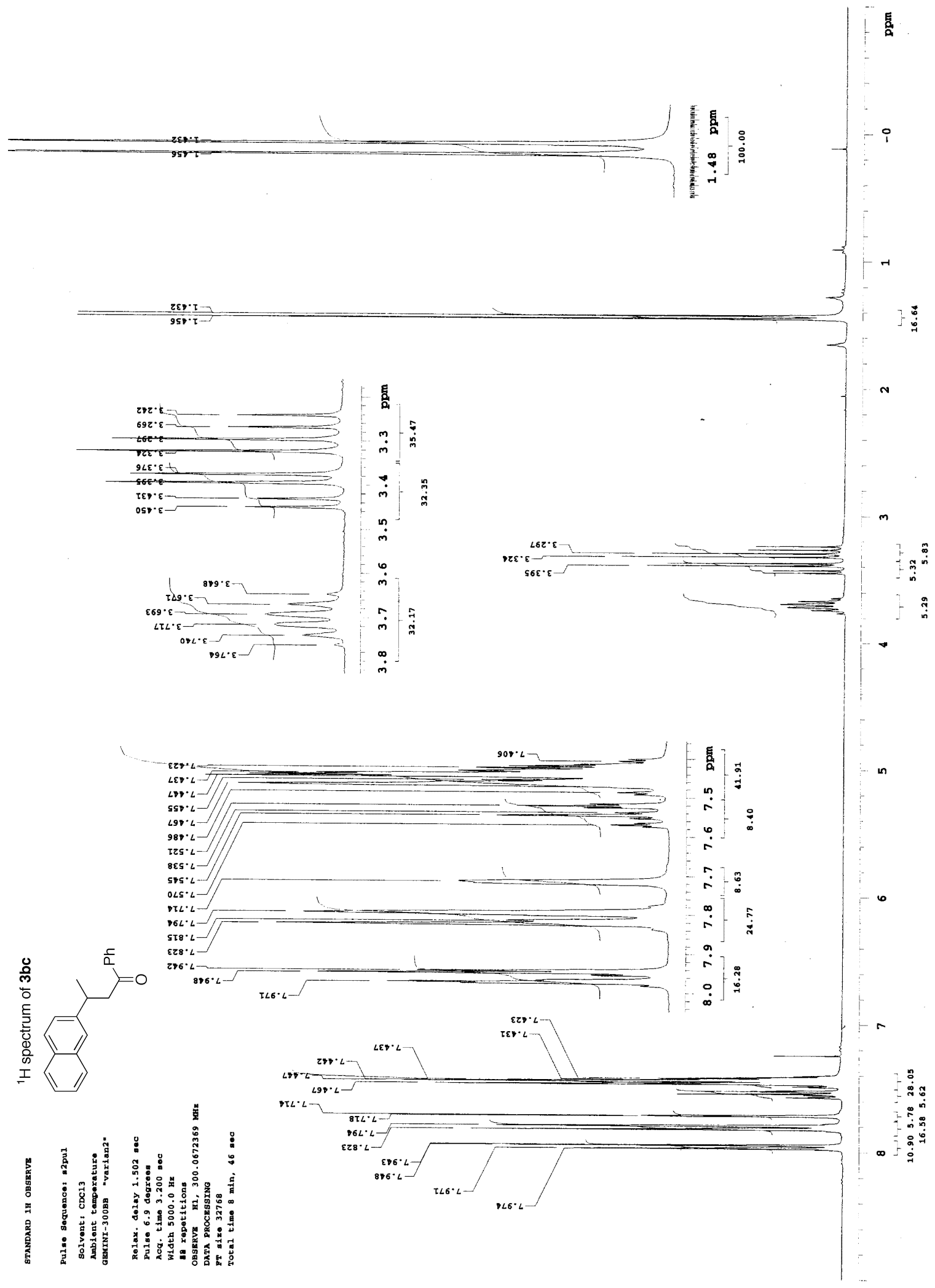




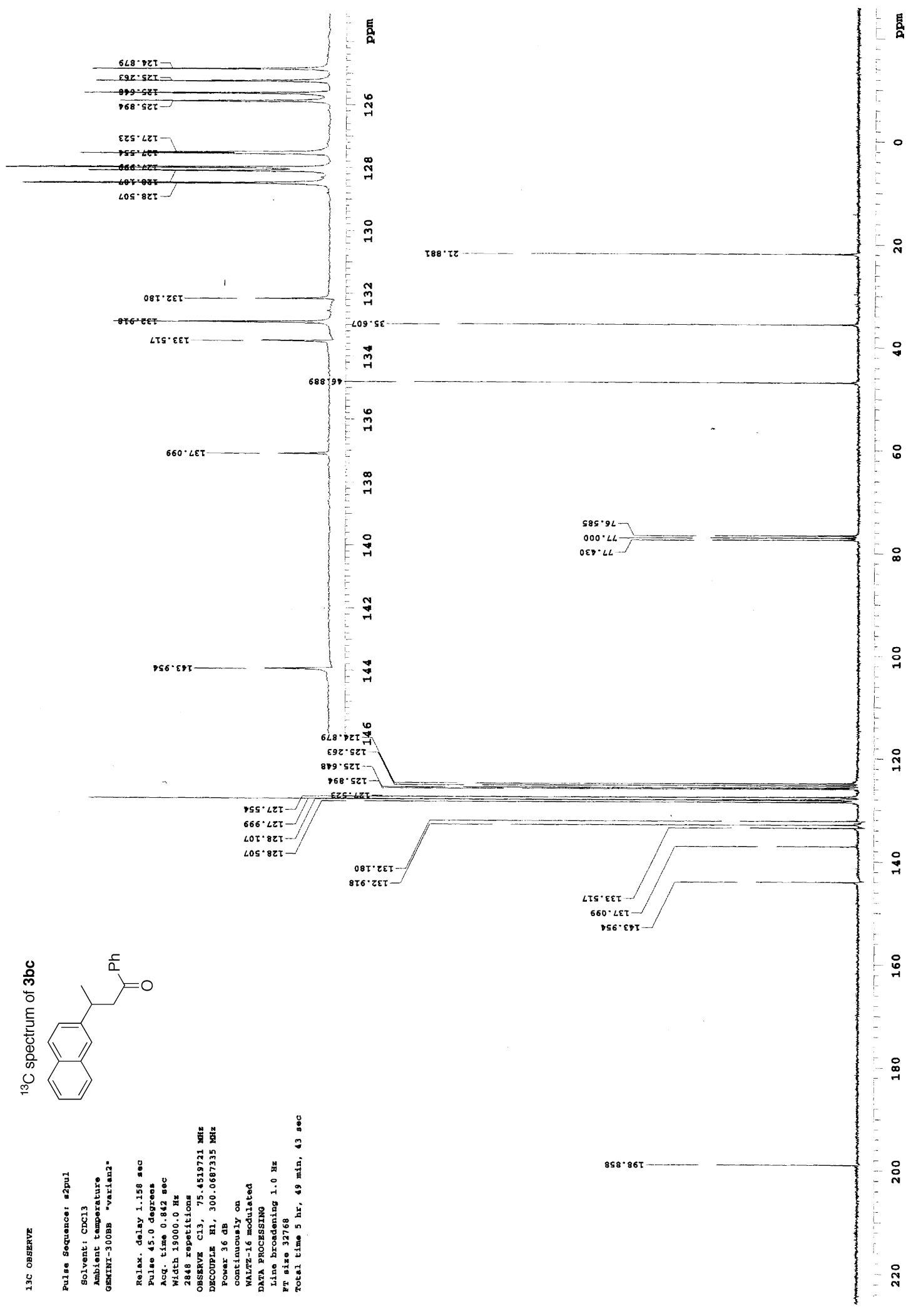




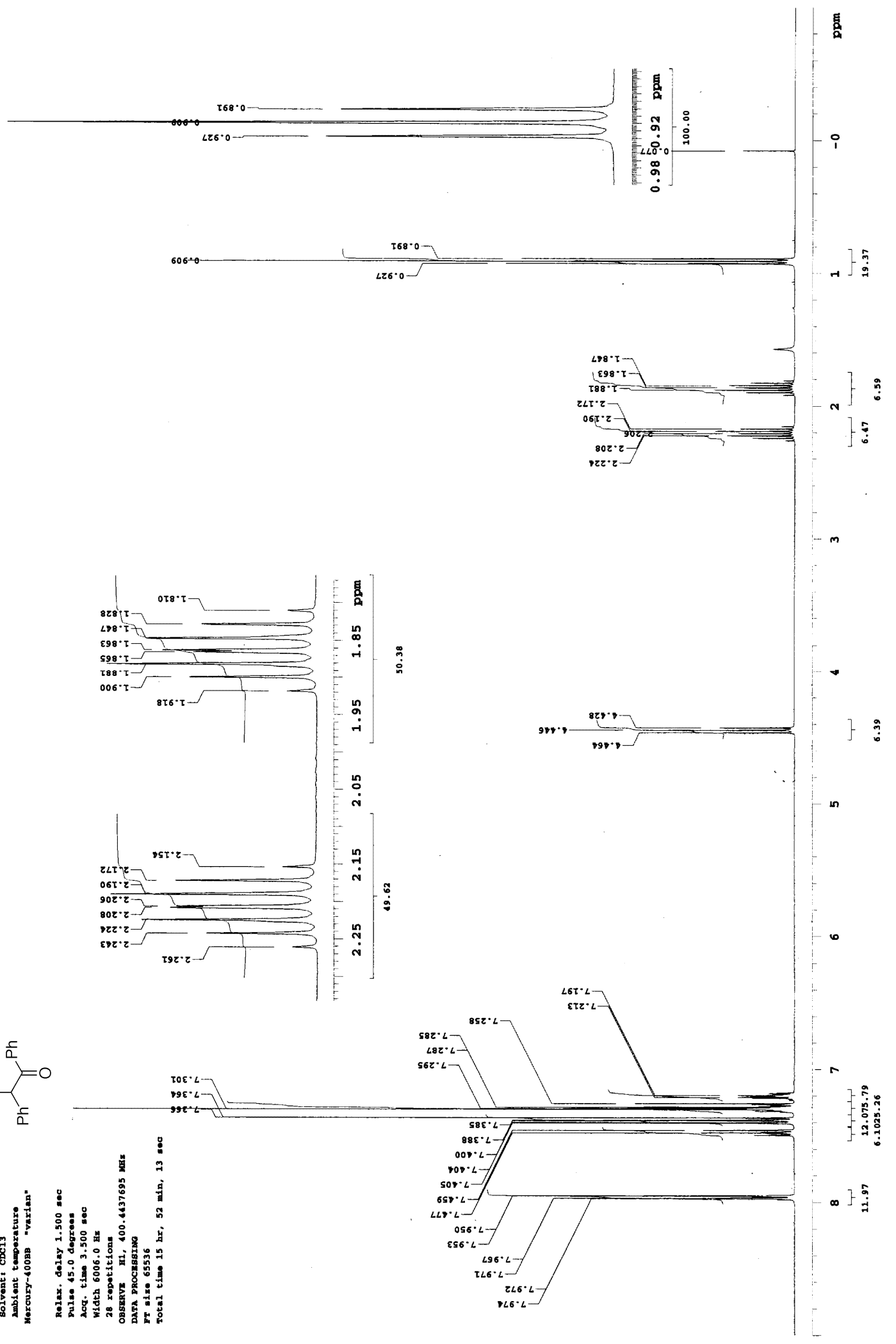




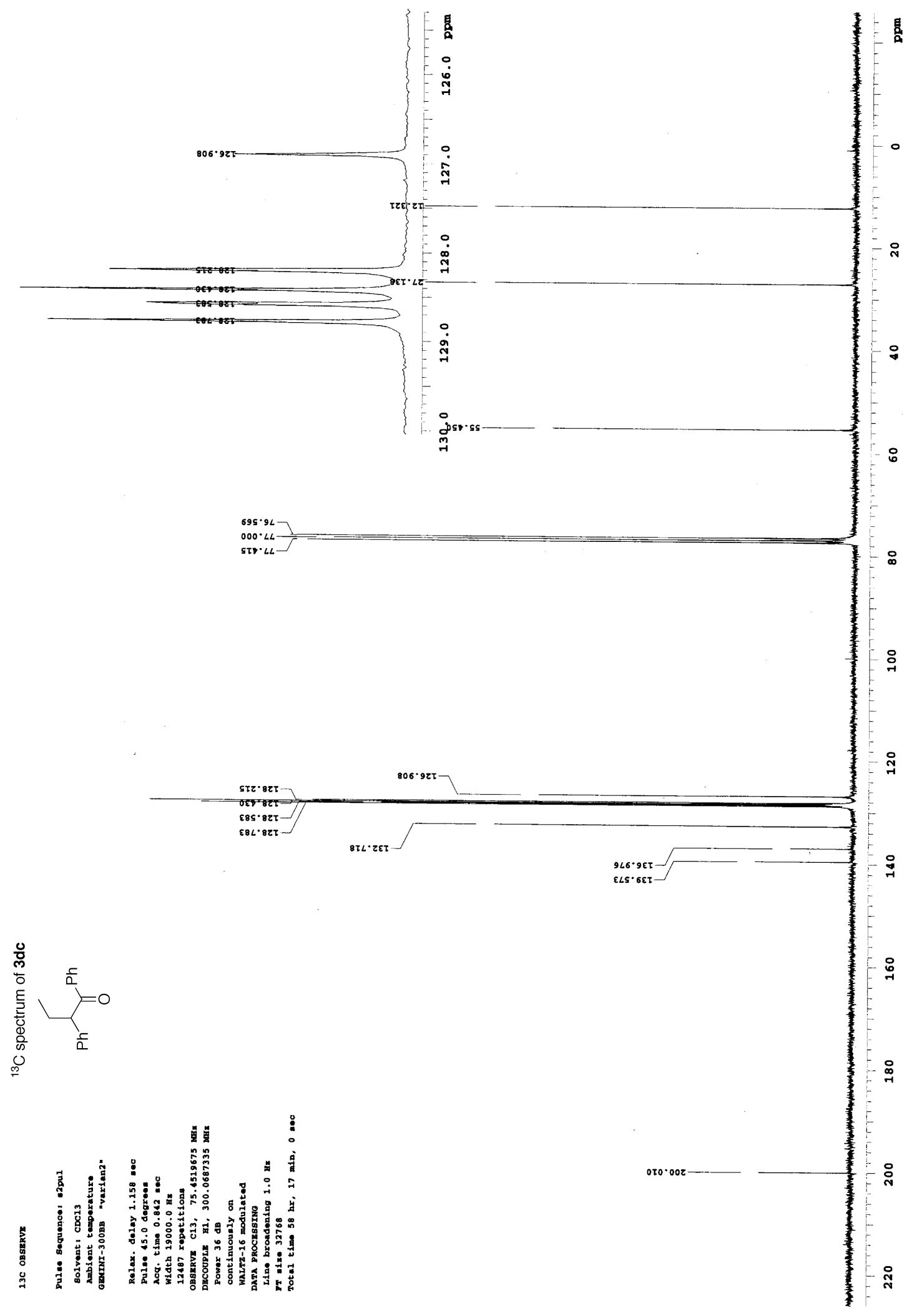




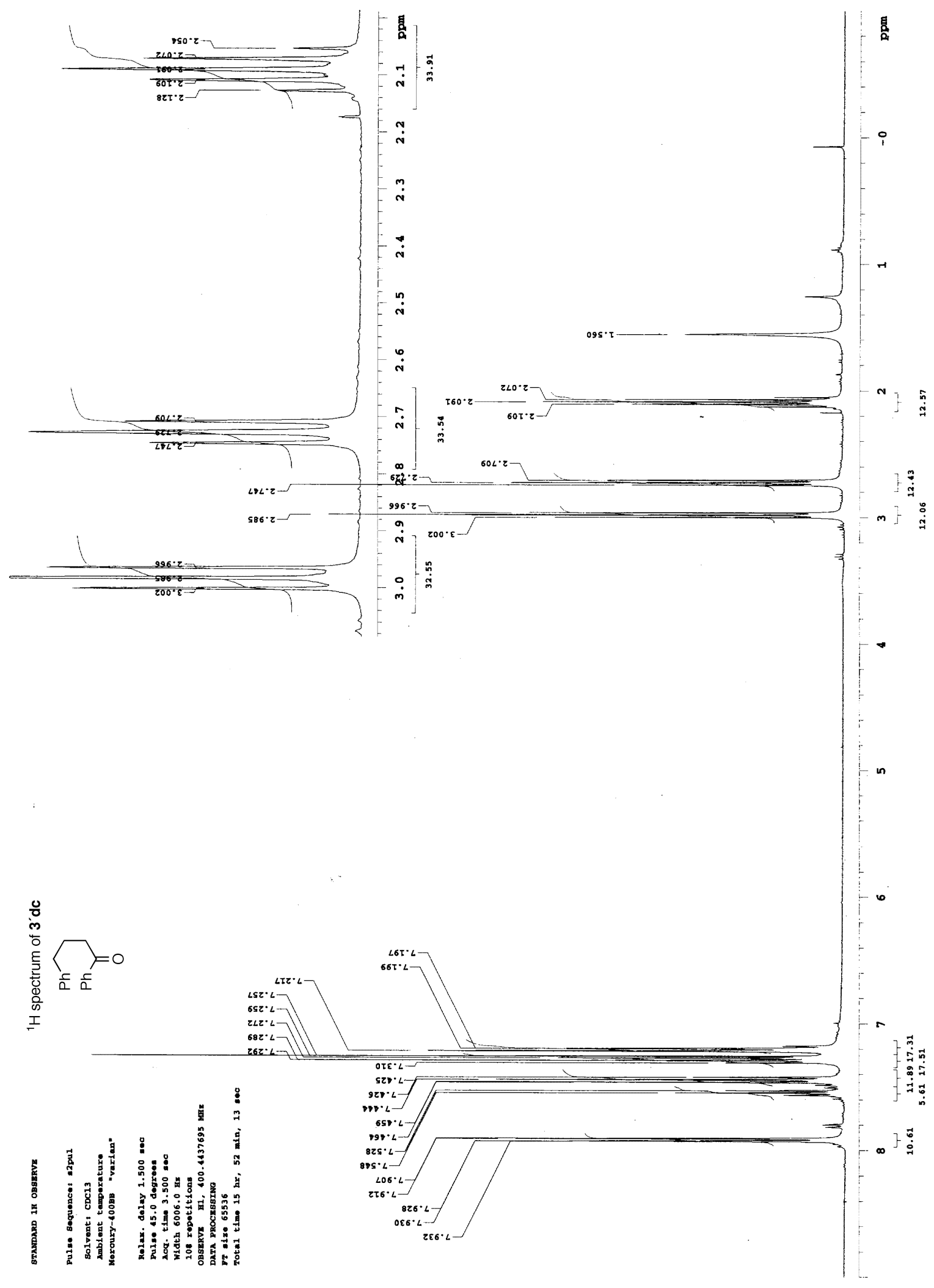




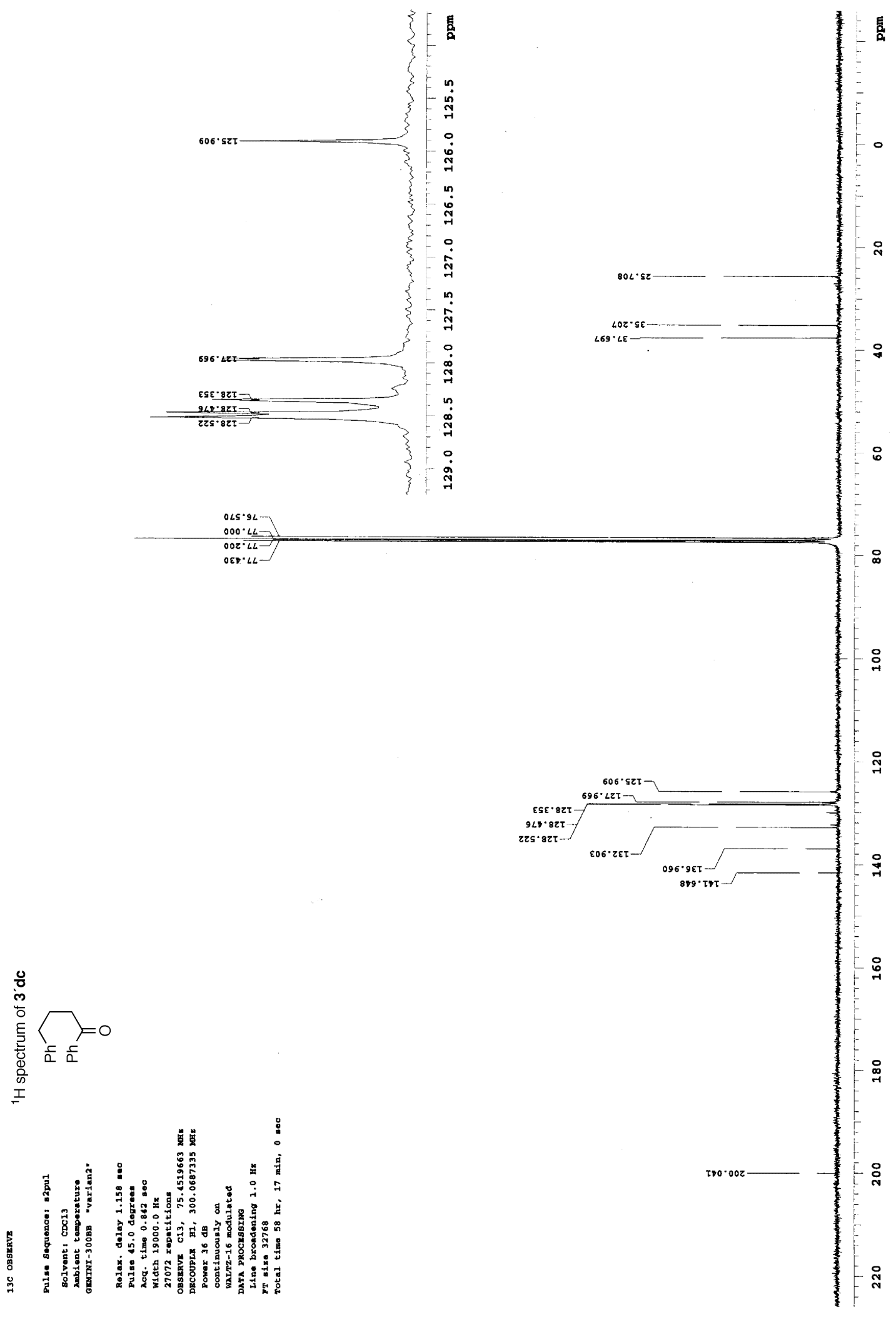




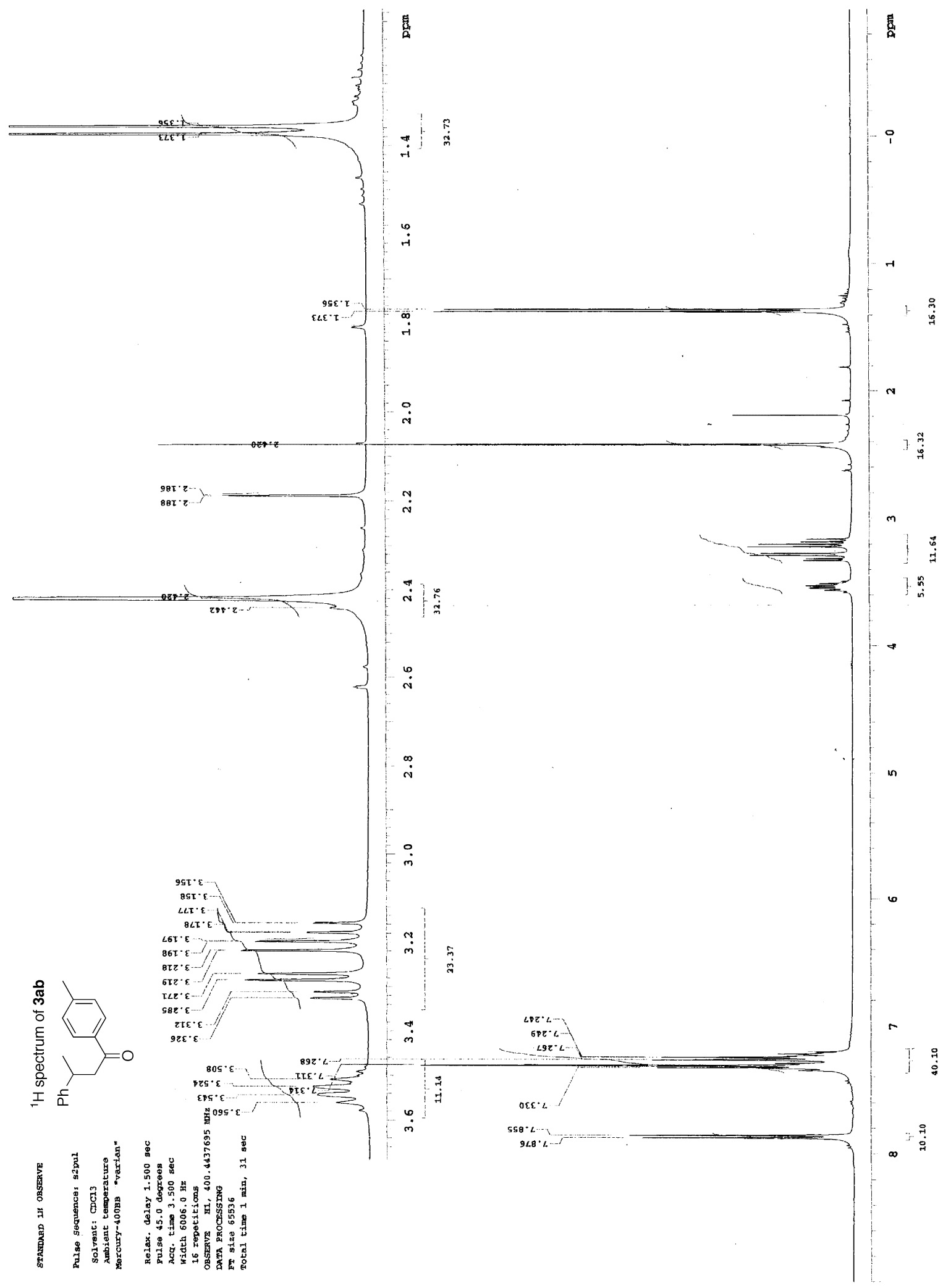




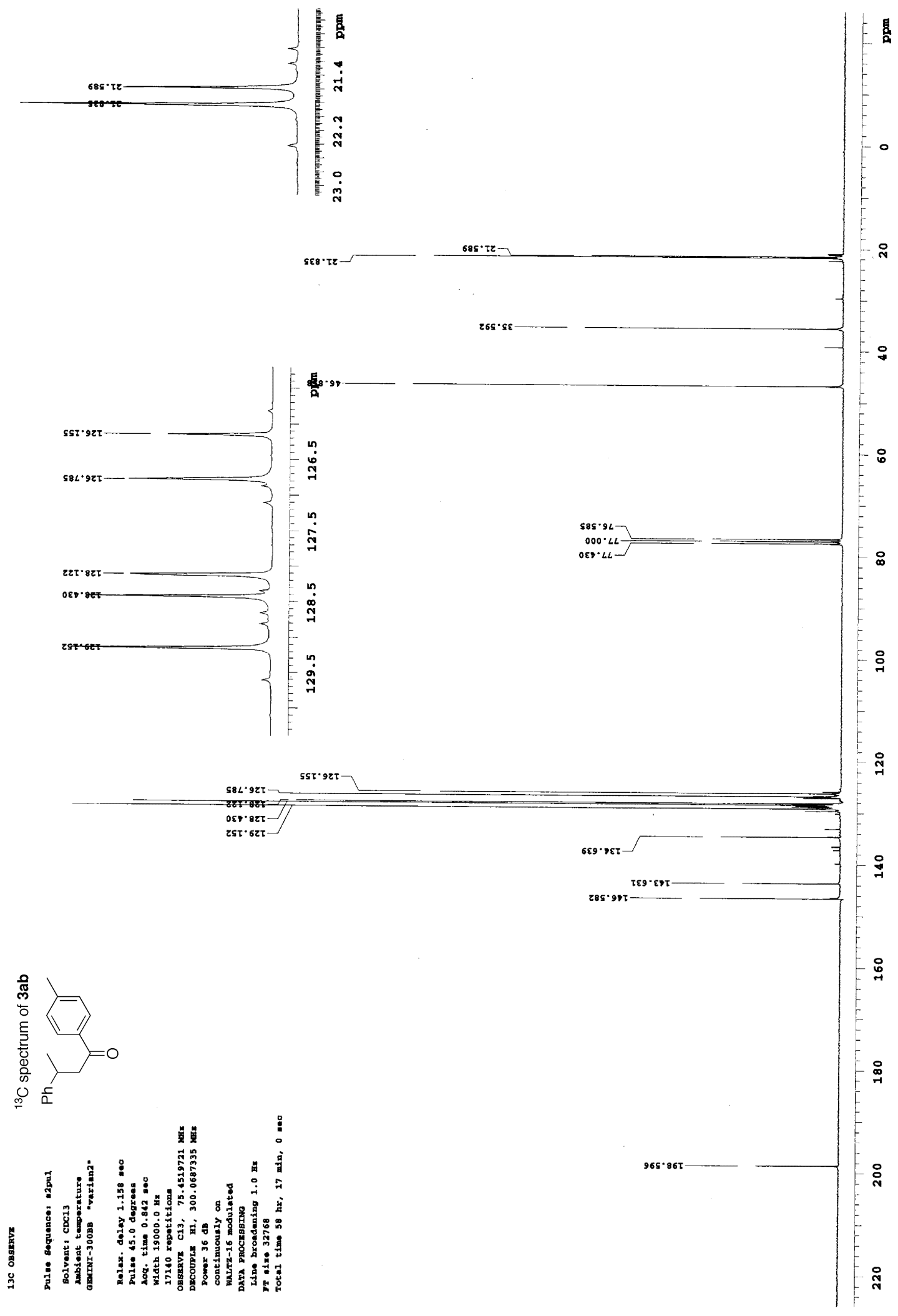




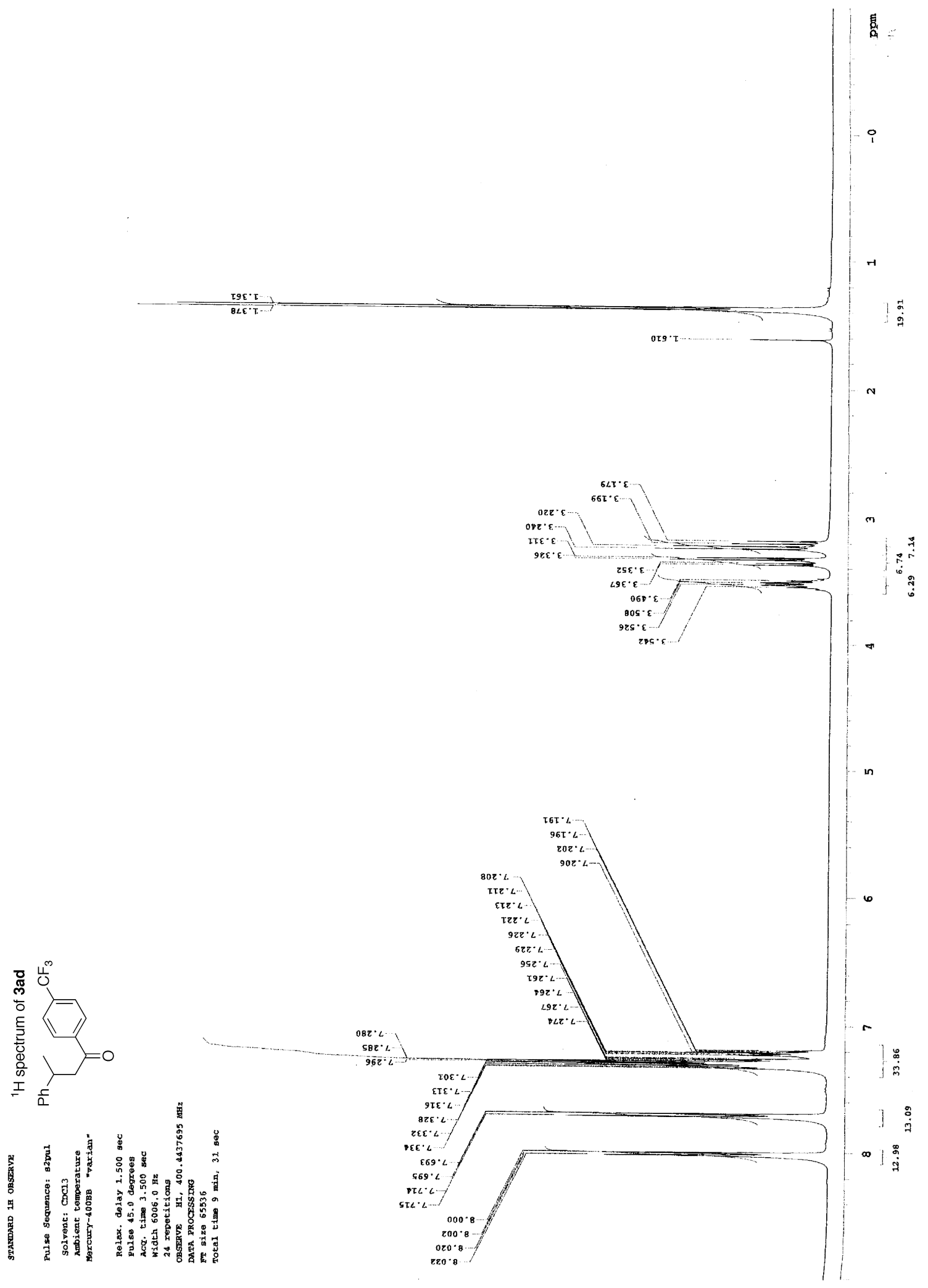




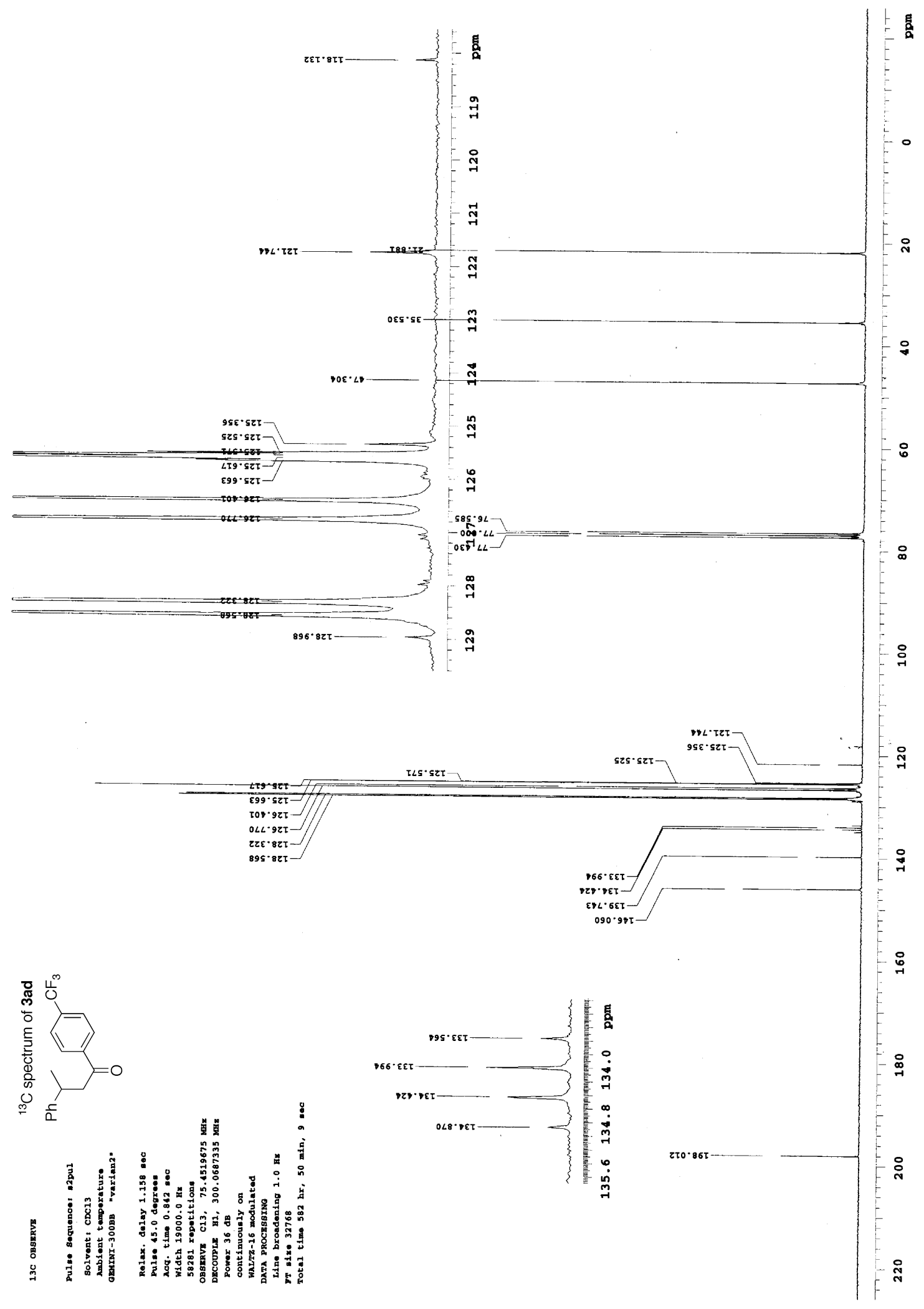




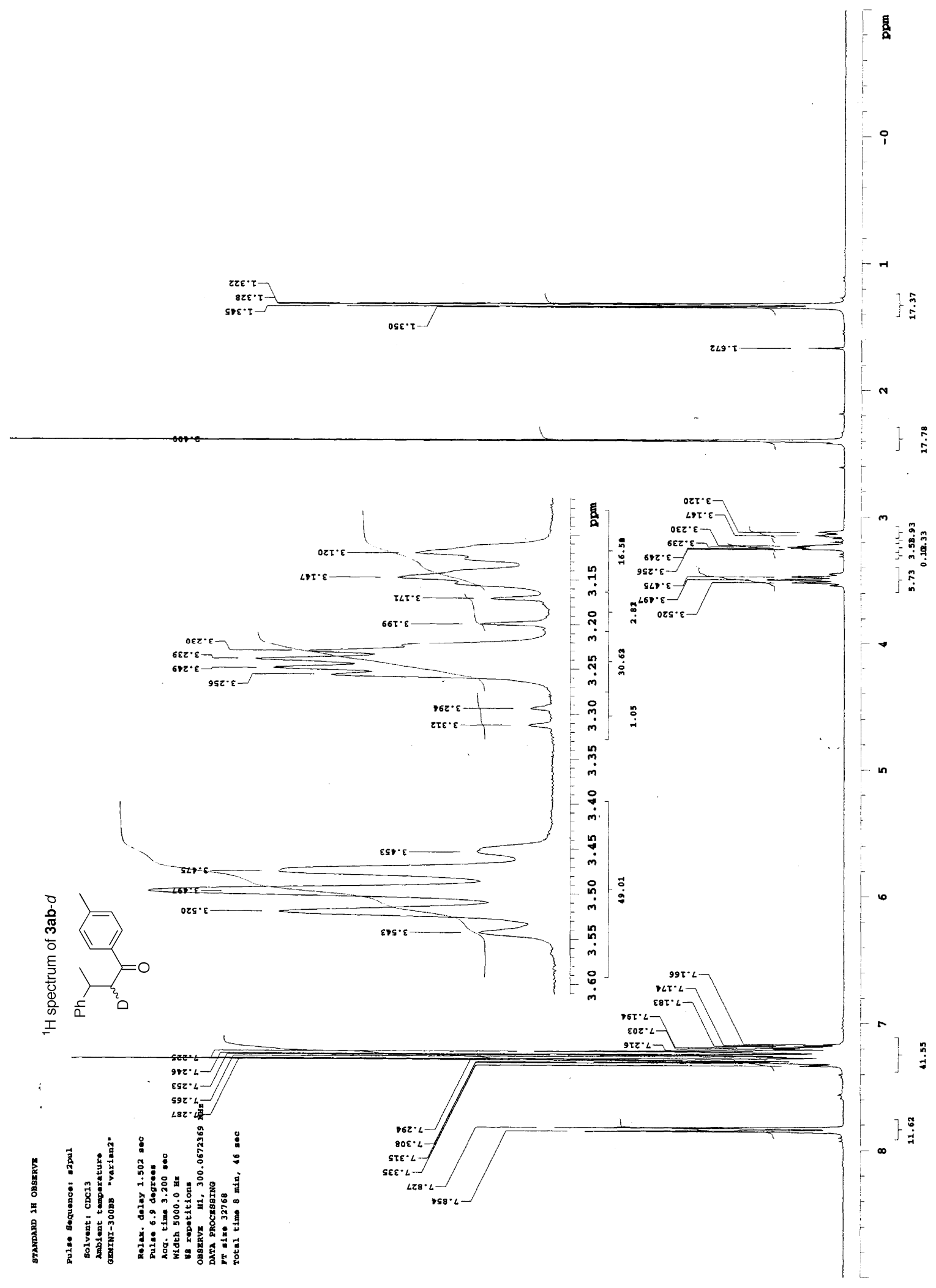




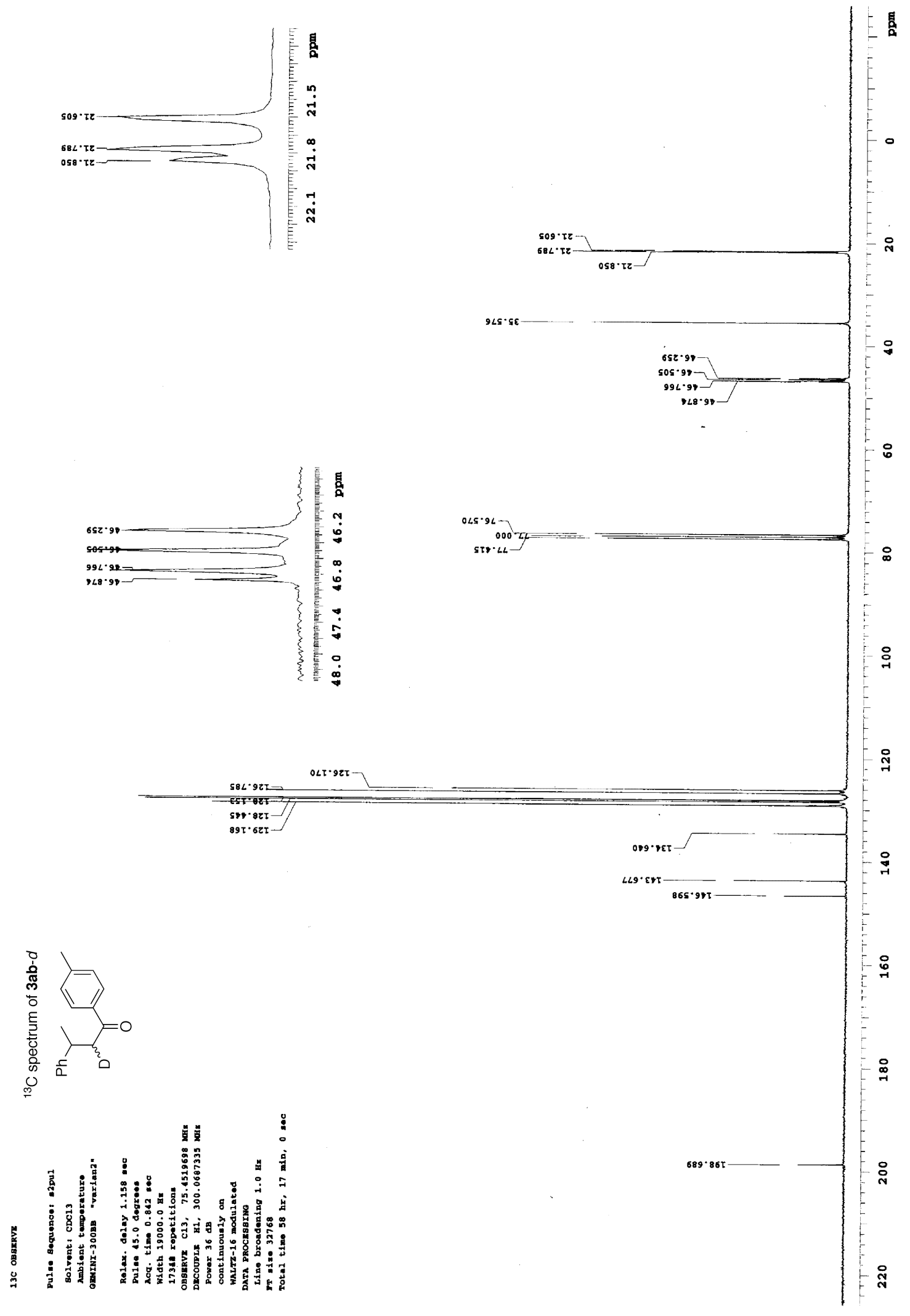




\section{References}

(1) (a) Hyatt, J. A.; Raynolds, P. W. Org. React. 1994, 45, 159. (b) Krepski, L.R.; Hassner, A. J. Org. Chem. 1978, 43, 2879. (c) Bak, D. A.; Brady, W. T. J. Org. Chem. 1979, $44,107$.

(2) Utimoto, K.; Tamura, M.; Sisido, K. Tetrahedron 1973, 29, 1169.

(3) Nemoto, H.; Miyata, J.; Yoshida, M.; Raku, N.; Fukumoto, K. J. Org. Chem. 1997, 62,7850 .

(4) Oi, S.; Moro, M.; Ito, H.; Honma, Y.; Miyano, S.; Inoue, Y. Tetrahedron 2002, 58, 91.

(5) Denmark, S. E.; Amishiro, N. J. Org. Chem. 2003, 68, 6997. 